\title{
On Polar Visibility Representations of Graphs
}

\author{
Joan P. Hutchinson* \\ Department of Mathematics and Computer Science \\ Macalester College \\ St. Paul, MN 55105, USA \\ hutchinson@macalester.edu
}

\begin{abstract}
We introduce polar visibility graphs, graphs whose vertices can be represented by arcs of concentric circles with adjacency determined by radial visibility including visibility through the origin. These graphs are more general than the well-studied bar-visibility graphs and are characterized here, when arcs are proper subsets of circles, as the graphs that embed on the plane with all but at most one cut-vertex on a common face or on the projective plane with all cut-vertices on a common face. We also characterize the graphs representable using full circles and arcs.
\end{abstract}

\section{Introduction}

Visibility graphs are now a well-established area of graph drawing [10. Much has been written about their importance and application; however, they continue to pique the imagination of mathematicians with their intrinsic appeal and intriguing questions [2]. There has been a natural progression from bar-visibility graphs (BVGs) [11|17] to rectangles [1]3|4|6], from bars with visibilities in the plane to those on the sphere and cylinder [12,13], on a (flat) torus 8], or on the Möbius band [5. These rectilinear representations are natural ones for most applications; however, we turn instead to the realm of polar representations with arcs of circles and radial visibility. In many ways circular representations and related polar coordinates are equally natural and in some contexts more applicable than rectilinear ones. With this change of perspective we can and do represent a class of graphs, larger than with bars in the plane, though ultimately constrained by the real projective plane. Thus with a planar representation of arcs of circles nonplanar graphs are drawn in a natural way, resulting in diagrams often reminiscent of time-exposed shots of the North Star and surrounding stars.

We introduce the layout of graphs as polar visibility graphs (PVGs) using arcs of concentric circles (arcs that are proper subsets of a circle) with radial visibility, including visibility through the origin, the center of all the concentric circles. These graphs, though arising naturally from visibility in the plane, correspond to graphs embedded on the (real) projective plane, the nonorientable surface of Euler characteristic 1. PVGs are characterized as the planar graphs that can be

* Research supported in part by NSA Grant \#MDA904-99-1-0069.

P. Mutzel, M. Jünger, and S. Leipert (Eds.): GD 2001, LNCS 2265, pp. 422-434, 2002.

(C) Springer-Verlag Berlin Heidelberg 2002 
drawn in the plane with all but at most one cut-vertex on a common face plus the graphs that can be embedded on the projective plane with all cut-vertices on a common face. We also consider the variation in which full circles are allowed along with arcs, and characterize the graphs so representable (CVGs) in terms of their block-cutpoint tree.

\section{Background}

Just as visibility wider than along a line is required for BVGs, we ask that radial visibility in PVGs be available through a nondegenerate cone. Define a (nondegenerate) cone in the plane to be a 4 -sided region of positive area with two opposite sides being arcs of circles, centered at the origin, and the other two sides, possibly intersecting, being radial line segments on lines through the origin. Thus, both $\{(r, \theta): 1 \leq r \leq 2,0 \leq \theta \leq \pi / 6\}$ and also

$\left\{(r, \theta): 0 \leq r \leq 1,0 \leq \theta \leq \frac{\pi}{6}\right.$ or $\left.\pi \leq \theta \leq \frac{7 \pi}{6}\right\}=\left\{(r, \theta):-1 \leq r \leq 1,0 \leq \theta \leq \frac{\pi}{6}\right\}$

are considered to be cones, respectively, not containing and containing the origin. Given a set of arcs, all centered at the origin, two of these $\operatorname{arcs} a_{1}$ and $a_{2}$ are said to be radially visible if there is a cone that intersects only these two arcs and whose two circular ends are subsets of the two arcs; the same definition holds for visibility between an arc and a circle and between two circles. A graph is called a polar visibility graph if its vertices can be represented by arcs, including endpoints, of circles centered at the origin, having pairwise disjoint relative interiors, so that two vertices are adjacent if and only if the corresponding arcs are radially visible; see Figure 1a below for a PVG representation of $K_{6}$. If this model is used, but without visibility through the origin, the graphs arising are one of the cylindrical types characterized in [13. Note that for a 2-connected graph (a graph without cut-vertices) there is no loss in taking arcs as proper subsets of circles since a full circle can be cut down to a smaller arc, leaving the same visibilities. Arcs in a PVG layout spanning more than half its circle will provide interesting variations, full circles even more. We use graph theoretic terminology as in [15], topological notions as in 9], and algorithmic ideas following the BVG presentation in [10].

Similarly a graph is called a circular visibility graph if its vertices can be represented by arcs and circles with radial visibility between arcs and circles determining edges as for PVGs. When possible we prefer, but do not require, arcs over circles; that is, in a layout we will decrease a circle to become a proper arc if no additional visibilities are introduced. We shall see that some planar and projective planar graphs with cut-vertices on an arbitrary number of faces are CVGs, but not PVGs, but that these faces must be nested appropriately. Figure 1b shows such a planar CVG. In that layout the inner circle contains one arc; if instead, it contained four mutually visible arcs, encircling the origin and forming a $K_{5}$, the example becomes a nonplanar CVG.

Note that in a PVG or CVG layout of a graph G, we may draw each arc and circle on a distinct circle, and we may take these circles to have radii $1,2, \ldots, n$ 

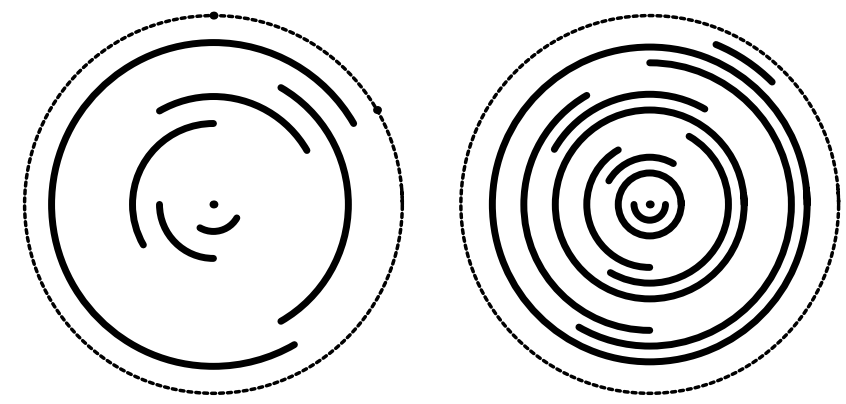

Fig. 1. a. $K_{6}$

b. A circular visibility layout

where $n=|V(G)|$. This naturally leads to another layout of the graph in a disk of radius $n+1$ and centered at the origin by inverting each circle and arc through the circle of radius $(n+1) / 2$. That is, each point with polar coordinates $(r, \theta), 0<r<n+1$, is mapped by the inversion to the point $(n+1-r, \theta)$. This inversion preserves circles, arcs, and the angles defining these arcs. If the original layout was $L$, we denote this inverted layout by $\mathbb{I}(L)$.

Recall that the (real) projective plane can be obtained by taking a circular disk and identifying opposite (or antipodal) points. Thus if we identify opposite points of the circle of radius $n+1$, we create a projective plane. Two arcs in $\mathbb{I}(L)$ (or an arc and a circle or two circles) that were previously radially visible in a cone, not containing the origin, are still radially visible, and a pair visible in a cone through the origin are now visible in a "generalized cone" that crosses the boundary of the projective plane, reemerging on the other side. The coordinates of such a generalized cone are given by $\left\{(r, \theta), r^{*} \leq r \leq n+\right.$ 1or $\left.-(n+1) \leq r \leq-s^{*}, \theta_{1} \leq \theta \leq \theta_{2}\right\}$ where $r^{*}$, s*, $\theta_{1}<\theta_{2}$ are constants, $0 \leq r^{*}, s^{*}<n+1$. In addition, the interior of no two of these new cones intersect. Fig. 2 a shows the inverted layout of $K_{6}$ on the projective plane with dashed lines indicating a conical area of visibility, and in $2 \mathrm{~b}$ we see an embedding of $K_{6}$ created by shrinking each arc to a vertex. The first proposition is then clear since each inverted arc and circle on the projective plane can be replaced by a single vertex. Then the visibility cones can each be shrunk and transformed to a set of nonintersecting edges on the projective plane.

Proposition 1. A PVG or a CVG embeds on the projective plane. Recall that a graph $G$ is said to embed on a surface $S$ if it can be drawn there without any edge crossings, and that each maximal connected component of $S \backslash\{V(G), E(G)\}$ is called a face of the embedding (we do not require that the faces be simply connected).

Theorem 1. A graph Gis a PVG if and only if either a) $G$ has an embedding in the plane with all but at most one cut-vertex on a common face, or else b) $G$ has an embedding on the projective plane with all cut-vertices on a common face. 

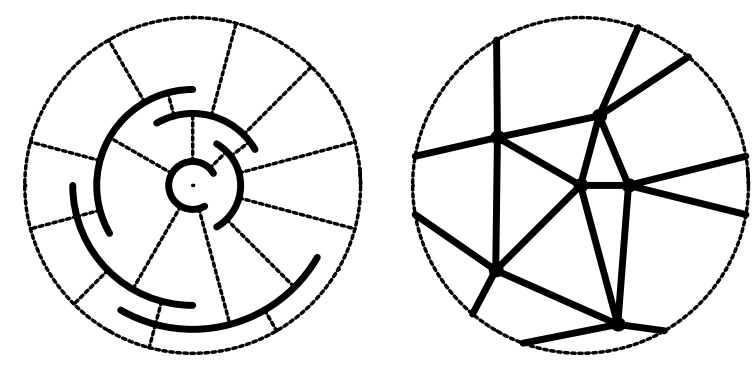

Fig. 2. a. $\mathbb{I}\left(K_{6}\right)$ and $\mathbb{I}\left(K_{6}\right)^{*}$ on the projective plane b. For $G=K_{6}, \mathbb{I}\left(L_{G}\right)=(\mathbb{I}(L))_{G}$

Note that condition (a) allows for the representation of planar graphs that are not BVGs; for example $K_{2,3}$ with three additional vertices of degree 1 appended, one each to a vertex of degree two, is a PVG. Similarly $K_{4}+4 e\left(K_{4}\right.$ plus a pendant vertex and edge at each vertex) is a PVG (see Fig. 3); these are the smallest graphs that are not BVGs. Condition (b) also allows for more planar graphs; for example, two vertices joined by three internally disjoint paths of length three (i.e., three edges each) plus six vertices of degree 1, each adjacent to a different vertex of degree two, satisfies (b), but not (a).

Every graph $G$ can be decomposed into its blocks and their connecting cutvertices (a block is either an edge or a 2-connected subgraph; see 15]), and these connections determine a tree, called the block-cutpoint tree of the graph, $\mathrm{BC}(G)$. This tree has a vertex for each block and for each cut-vertex of $G$, and two vertices of $\mathrm{BC}(G)$ are adjacent if and only if they correspond to an incident cut-vertex and block. We call a block planar if it represents a planar graph.

Theorem 2. A graph $G$ is a $C V G$ if and only if $\mathrm{BC}(G)$ consists of a path $P=\left(e_{1}, e_{2}, \ldots, e_{2 k+1}\right), k \geq 0$, with $e_{2 i}$ representing a planar block, $i=1, \ldots, k$, so that

1. a) $e_{1}$ is also incident with one additional (nonempty) block representing a (2-connected) projective planar graph, or

b) $e_{1}$ is also incident with one or more (nonempty) planar blocks, and

2. $e_{2 k+1}$ is also incident with an arbitrary tree structure $T$ so that $T \cup\left\{e_{2 k+1}\right\}$

represents a planar graph that can be drawn in the plane with all cut-vertices, except possibly for that representing $e_{2 k+1}$, on a common face.

When $k=0$, these conditions reduce to those of Theorem 11. On the other hand, it may be that each cut-vertex of $G$, represented by $e_{1}, e_{3}, \ldots, e_{2 k+1}$, lies on a different face, as in Figure 1b. This example is the first of an infinite family of CVGs with an increasing number of cut-vertices, all on different faces; the family is obtained by nesting repeatedly the same pattern of arcs and circles. Most of the details of the proofs of Theorems 1 and 2 are included below.

As described in [10, planar layouts and the block-cutpoint tree of a graph can be determined in linear time. Projective planar graphs can also be recognized 
and embedded in linear time 7. It can quickly be determined whether all cutvertices of a graph lie on a common simple cycle and, if so, whether there is an embedding in either surface in which this cycle bounds a face. The proofs of Theorems 1 and 2 together with standard BVG algorithms, lead to a $\mathcal{O}\left(\mathrm{NE}^{2}\right)=$ $\mathcal{O}\left(N^{3}\right)$ time algorithm for laying out a PVG $G$ with $\mathrm{N}$ vertices and E edges, given an embedding of $G$ in the projective plane as a rotation scheme (defined below), as in 79 .

\section{Main Results on PVGs}

We develop theory that will also allow extension to CVGs. We focus on simple graphs and their characterizations as in Theorems 1 and 2 Thus we say that two arcs are radially visible if there is at least one maximal cone providing mutual visibility; however, we can also obtain more precise results by keeping track of multiple and even self-visibility between arcs and circles.

First we need more precise topological and geometric definitions. Consider a PVG or CVG layout $L$ of a graph $G$ and its inverse layout on the projective plane, $\mathbb{I}(L)$. We let $L^{*}$ (respectively, $\mathbb{I}(L)^{*}$ ) denote the visibility depiction obtained by shrinking each maximal visibility cone of $L$ (resp., $\mathbb{I}(L))$ to a distinct line segment by reducing its angles $b_{1} \leq \theta \leq b_{2}$ to some constant $\theta=b, b_{1}<b<b_{2}$; strict inequality ensures distinct visibility segments. For $G=K_{6}, \mathbb{I}(L)^{*}$ is shown in Fig. 2a.

Also let $L_{G}$ (resp., $\left.(\mathbb{I}(L))_{G}\right)$ denote the graph obtained from $L^{*}$ (resp., $\left.\mathbb{I}(L)^{*}\right)$ by shrinking each arc to a vertex, consisting of one point, and transforming each visibility line segment to an edge that intersects no other edge except possibly at the origin (resp., an edge that intersects no other edge on the projective plane). If $L^{*}$ or $\mathbb{I}(L)^{*}$ contains a circle, it is replaced by a point as vertex. Thus $(\mathbb{I}(L))_{G}$ is a graph embedded on the projective plane, see Fig. 2b. Note that $L^{*}$, $\mathbb{I}(L)^{*}, \mathbb{I}\left(L_{G}\right)$, and $(\mathbb{I}(L))_{G}$ have visibility segments and edges for each distinct, maximal visibility cone so that multiple edges and loops may be present in these depictions; however, a pair of multiple edges will not form an embedded digon with empty interior.

Note that the complement of the arcs, circles, and lines of $L^{*}$ divide up the plane into faces; similarly $\mathbb{I}(L)^{*}$ divides up the projective plane. One face of $L^{*}$ is the exterior face, possibly containing the origin; this exterior face is the one in which most cut-vertices of a PVG and their blocks can be placed. We say that an arc or circle of a layout $L$ lies on the exterior face if it lies on the exterior face of $L^{*}$. We use the following combinatorial description of an embedded graph and of a PVG or CVG layout. If a graph is embedded on any surface, then for each vertex there is naturally defined a cyclic rotation of its neighbors, given by the order, say clockwise, of its edges in the embedding; such a collection of rotations, one for each vertex, is called a rotation scheme. (See, for example, [16] where it is shown that an embedding is equivalent to a rotation scheme.) Such a description is generally used for algorithms on embedded graphs [9]. Similarly, given a PVG layout $L$ in the plane and its inverse layout $\mathbb{I}(L)$ in the projective 
plane, one can define the arc-rotation scheme to be the set of cyclic rotations of neighbors about each arc of its visibilities to other arcs; note that the rotations at the $\operatorname{arcs}$ of $L$ and of $\mathbb{I}(L)$ are inverses of each other. We say that an embedding of a PVG graph $G$ in the plane or on the projective plane and its polar visibility layout $L$ are equivalent if the arc-rotation scheme of $\mathbb{I}(L)$, when translated into a set of vertex-neighbor cycles, yields the rotation scheme of the embedded graph; see Figs. 1, 2. Given a circle in a CVG layout $L$ or $\mathbb{I}(L)$, the neighbors divide into two cyclic rotations of the inner and outer visibilities, called the circlerotation scheme. Then a drawing of a CVG and its layout $L$ are equivalent if the arc/circle-rotation schemes of $\mathbb{I}(L)$ agree with those of the embedded graph.

It is not hard to see the following, by bending or straightening corresponding BVG and PVG layouts.

Proposition 2. A connected graph has a PVG layout with no visibilities through the origin if and only if the graph is a $B V G$.

A PVG layout with no visibilities through the origin contains arcs in sectors, alternating about the origin, so that some can be reflected through the origin, leaving the layout in two quadrants, and this can be straightened to form a BVG.

Of course there are planar graphs with layouts as PVGs including visibilities through the origin and with cut-vertices represented on the exterior face. Note that whenever there are visibilities through the origin in a layout $L$, then the equivalent graph $(\mathbb{I}(L))_{G}$ is embedded on the projective plane. It turns out that in some PVG layouts there is a (sneaky) hiding place for a cut-vertex and its connecting blocks, but the resulting graphs turn out to be planar. In a PVG layout we call an arc $a^{*}$ a long arc if its angular span is greater than $\pi$. Suppose $a^{*}=\left\{\left(r^{*}, \theta\right), 0 \leq \theta \leq \pi+x\right\}$, for some $0<x<\pi$. Then the cone defined by $C\left(a^{*}\right)=\left\{(r, \theta),-r^{*} \leq r \leq r^{*}, 0 \leq \theta \leq x\right\}$ is an area in which interior arcs can see the arc $a^{*}$ and possibly no others; see Fig. 3

In preparation for CVG layouts, we require special PVG layouts. Let $a^{*}$ be a long-arc at radius 1 , spanning $\theta_{1} \leq \theta \leq \theta_{1}+\pi+x$ for some $x>0$. Arcs $a^{*}$ and $b^{*}$ are called a long-arc pair at the origin if they are mutually visible, together they span $2 \pi$, and if $b^{*}$ lies at radius $r^{*}>1$, no arcs intersect the long-arc cone $\left\{(r, \theta): 0 \leq r<r^{*}, \theta_{1}+\pi+x<\theta<\theta_{1}+2 \pi\right\}$. (For example, when $r^{*}=2$, no arcs can meet the designated cone.) Similarly if $a^{*}$ is a long-arc at the outermost radius $n=|V(G)|$, spanning $\theta_{2} \leq \theta \leq \theta_{2}+\pi+y$ for some $y>0$, then $a^{*}$ and $b^{*}$ are a long-arc pair at infinity if they are mutually visible, together span $2 \pi$, and if $b^{*}$ lies at radius $r^{*}<n$, no arcs intersect the long-arc cone $\left\{(r, \theta): r^{*}<r<n, \theta_{2}+\pi+y<\theta<\theta_{2}+2 \pi\right\}$; see Figs. 1a, 3. Notice that in long-arc pairs the long arc at radius 1 or at radius $n$ could be extended to form a full circle without changing visibilities.

Here are the building-block results needed for the PVG characterization.

Proposition 3. Let $G$ be laid out as a PVG L including a long arc $a^{*}$ that represents a cut-vertex $x^{*}$, not lying on the exterior face, and let $B$ be a block of $G$ incident with $x^{*}$ and whose representation lies within $C\left(a^{*}\right)$ in $L$. Then $G$ is 


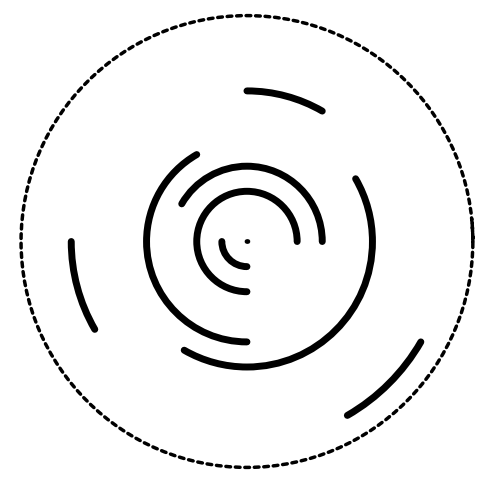

Fig. 3. $K_{4}+4 \mathrm{e}$.

a planar graph and can be drawn in the plane with one face including all vertices whose arcs lie on the exterior face of $L$.

Proof. The proof consists of observing that in $\mathbb{I}(L)$ and in $(\mathbb{I}(L))_{G}$ the representation of a block $B$ incident with $x^{*}$ lies within a (noncontractible) sector of the projective plane that divides the space into two contractible (planar) regions.

The next result is the necessary topological argument needed to characterize PVGs; it is a contraction proof similar to that of [14] and [8]. This result is carried out for multigraphs, those embedded with no digon face with empty interior except for two special faces. For these graphs we achieve layouts with a one-to-one correspondence between distinct, maximal visibility cones and edges of $G$. If $x$ is a vertex of a PVG, we let $a_{x}$ denote its arc in the layout, and conversely $x_{a}$ is the vertex corresponding to an arc $a$.

Proposition 4. (i) Let $G$ be a loopless 2-connected plane multigraph, let $F$ be a face in the embedding, and let $c$ be a vertex of $G$. Suppose $G$ has at most two digon faces, possibly $F$ and, when $c$ does not lie on $F$, possibly one incident with $c$. Then $G^{\prime}=G$ plus a loop at $c$ has a $P V G$ layout $L^{\prime}$ in which all vertices of $F$ are represented on the exterior face of $L^{\prime}$ and $\left(a_{c}, a_{d}\right)$ is a long-arc pair at the origin for some neighbor $d$ of $c$. In addition, $G^{\prime}$ has an embedding on the projective plane that is equivalent to $L^{\prime}$.

(ii) Let $G$ be a loopless 2-connected plane multigraph with $v_{1}$ and $v_{2}$ designated, distinct vertices and with no digon face. Then $G^{\prime}=G$ plus a loop at $v_{2}$ has a PVG layout $L^{\prime}$ with $v_{i}$ represented by arc $a_{i}, i=1,2$, with $\left(a_{1}, b_{1}\right)$ a longarc pair at infinity, and with $\left(a_{2}, b_{2}\right)$ a long-arc pair at the origin, where for $i=1$ and 2, arc $b_{i}$ corresponds to some neighbor of $v_{i}$. Also $G^{\prime}$ has an embedding on the projective plane that is equivalent to $L^{\prime}$.

(iii) Let $G$ be a loopless 2-connected multigraph with a 2-cell embedding on the projective plane, with $F$ a face in the embedding, and with no digon face except possibly for $F$. Then $G$ has a PVG layout $L$ that is equivalent to the embedding of $G$ with exterior face corresponding to $F$. 
(iv) Let $G$ be a loopless 2-connected multigraph with a 2-cell embedding on the projective plane, with no digon face, and with $v_{1}$ a designated vertex. Then $G$ has a PVG layout $L$, equivalent to the embedding of $G$, in which $v_{1}$ is represented by arc $a_{1}$ with $\left(a_{1}, b_{1}\right)$ a long-arc pair at infinity and with arc $b_{1}$ corresponding to some neighbor of $v_{1}$.

Proof. Sketch of proof of (i). For most cases (when $c$ does not lie on $F$ ), the proof is by induction on $n$.

We can always find a nonloop, nonmultiple edge $e=(x, y)$ of $G$ so that $G$ with $e$ contracted, $G / e$, is 2-connected, loopless, embedded on the plane, and $F$ is still bounded by at least two edges. $G / e$ satisfies the inductive hypothesis and so has PVG layout $L_{e}$, equivalent to an embedding of $G / e$ plus a loop on the projective plane. If the contraction combines vertices $x$ and $y$ into new vertex $x^{*}$, let $a^{*}$ be its representation in $L_{e}$, at say radius $r$. Because the embeddings of $\mathrm{G} / \mathrm{e}$ and $L_{e}$ are equivalent, the lines of visibility to arcs representing vertices adjacent to $x$ in $G$ are consecutive in the rotation of visibility lines about $a^{*}$ in $L_{e}$. Then, when $a^{*}$ is not one of the special long arcs at the origin, it can be replaced by two $\operatorname{arcs} a_{x}$ and $a_{y}$ at radii $r-0.5$ and $r+0.5$ (or vice versa), representing vertices $x$ and $y$ of $G$, so that their visibilities give all edges incident with $\mathrm{x}$ and $\mathrm{y}$ and preserve the arc-rotations at $x$ and $y$ in $G$; see Fig 4 . This alteration gives the desired PVG layout $L$ for $G$. The argument is similar, though a bit more intricate, when $a^{*}$ is part of the long-arc pair at the origin. The proofs for (ii-iv) are analogous.

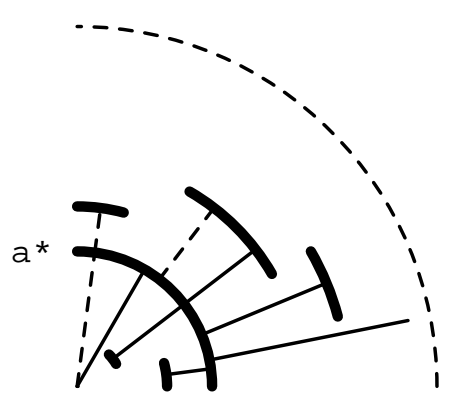

Fig. 4. An arc and its neighbors.

We then obtain the following.

Proposition 5. If $G$ has a $P V G$ layout $L$, then the embedding $(\mathbb{I}(L))_{G}$ of $G$ on the projective plane has cut-vertices on at most two faces. If the embedding has cut-vertices on two faces, then on one face there is only one cut-vertex, represented in $L$ by a long arc. 
Corollary 1. If $G$ has a $P V G$ layout $L$ with a long arc $a^{*}$, representing a cutvertex $x^{*}$ and not lying on the exterior face, then $G$ has a planar embedding with all cut-vertices except for $x^{*}$ lying on a common face.

Theorem 3. A simple planar graph $G$ has a $P V G$ representation if it has a planar embedding with all but at most one cut-vertex on a common face.

Proof. (Sketch) Assume that $G$ is not a BVG and so can be drawn in the plane with cut-vertices lying on the exterior face $F_{1}$ and an additional cut-vertex $c$ lying on $F_{2} \neq F_{1}$. Consider the block-cutpoint tree $\mathrm{BC}(G)$ of $G ; c$ may lie on several blocks, but at least one, call it $B_{0}$, contains a cut-vertex $c^{\prime} \neq c$ lying on $F_{1}$. Both of the faces $F_{i}$ are bounded by a facial walk $W_{i}$, and each $W_{i}$ contains a unique simple subcycle $C_{i}$, lying in $B_{0}$ and containing $c^{\prime}$ and $c$, respectively. If $G$ has cut vertices $c_{1}, \ldots, c_{i}$ lying on $F_{1}$, we label the blocks other than $B_{0}$ incident with $c_{1}, \ldots, c_{i} B_{1}, B_{2}, \ldots, B_{j}$, and the blocks $D_{1}, \ldots, D_{k}$ incident with c. Then we prove by induction on $j$ that there is a PVG layout $L$ of $G$ with $F_{1}$ represented by the exterior face of $L$, with $\left(a_{c}, a_{d}\right)$ a long-arc pair at the origin for some neighbor $d$ of $c$, and with the blocks incident with c represented within $C\left(a_{c}\right)$.

Theorem 4. If a simple graph has an embedding on the projective plane with all cut-vertices on a common face, then it is a PVG.

Proof. Let $G$ have an embedding on the projective plane $P$ with all cut-vertices on a common face $F$. We prove by induction on $n=|V(G)|$ that $G$ has a PVG layout with arcs representing cut-vertices on the exterior face and with its embedding equivalent to that of $G$. When $n<5$, the graph has a BVG layout and so a PVG one by Prop. 2, each such graph containing a cycle also has a 2-cell embedding on the projective plane and an equivalent PVG layout.

If $G$ has no cut-vertex, then we apply Prop. 4(iii) for graphs on the projective plane to get the PVG layout of $G$.

If $G$ has a cut-vertex, we consider the block-cutpoint tree $T$ of $G$, and, if possible, let $c$ be a cut-vertex incident with a leaf of $T$ with that leaf-block planar and embedded in a contractible region of $P$; call this block $B$. Deleting the vertices and edges of $B \backslash\{c\}$ leaves $G^{\prime}$ on the projective plane with face $F$ now a face $F^{\prime}$, containing all remaining cut-vertices. By induction $G^{\prime}$ has a PVG layout $L^{\prime}$ that is equivalent to $G^{\prime}$ and with exterior face representing $F^{\prime}$. Then there is a BVG layout of $B$ with the bar representing $c$ bottommost and extending the width of the layout, and by Prop. $2 B$ has a corresponding PVG layout $L_{B}$. Then $a_{c}$ in $L_{B}$ can be inserted as a subarc of $a_{c}$ on the exterior face of $L^{\prime}$ so that $L_{B}$ together with $L^{\prime}$ gives the desired layout of $G$.

Otherwise every leaf-block $B$ is embedded in a noncontractible region of $P$ and contains a noncontractible cycle in its embedding. If blocks $B$ and $B^{\prime}$ are two such leaves, they must intersect at a cut-vertex $c$ since every pair of noncontractible cycles on $P$ intersects. If there are additional blocks, there are additional leaves which must also all meet at $c$ so that $T$ is a star $K_{1, i}$ with the 
non-leaf vertex of $T$ representing $c$, the only cut-vertex of $G$, and each block is embedded in a wedge of $P$, all wedges meeting at, say, the origin. Such a graph is planar with one cut-vertex $c$ and so by Theorem 3 is a PVG.

Proof. of Theorem 1 for simple graphs. By Theorems 3 and 4 the graphs described are PVGs. Conversely if $L$ is a layout of a PVG $G$, then $G$ has an embedding on the projective plane by Prop. 1 with embedding $(\mathbb{I}(L))_{G}$. If $L$ has no visibility through the origin, then by Prop. $2 G$ is a BVG and so embeds in the plane with all cut-vertices on a common face. Otherwise, if $L$ contains a long arc, satisfying the conditions of Cor.1, then $G$ embeds in the plane with all but one cut-vertex on a common face. Otherwise $G$ embeds in the projective plane with all cut-vertices on a common face by Prop 5

\section{Results on CVGs}

As the example in Fig. $2 \mathrm{~b}$ and its extensions demonstrate, cut-vertices on many faces can be achieved using circles in layouts. We characterize CVGs in this section, as given in Theorem 2

Suppose $G$ has a layout $L$ with circles $c_{1}, c_{2}, \ldots, c_{k}$ at radii $r_{1}<r_{2}<\cdots<r_{k}$ and with no circle replaceable by an arc so that the same visibilities are achieved. The circles $c_{i}$ divide up the plane into annular regions and one projective planar region; note that neither the interior of $c_{1}$, denoted $\operatorname{int}\left(c_{1}\right)$, nor the exterior of $c_{k}, \operatorname{ext}\left(c_{k}\right)$, is empty in $L$ since neither circle can be replaced by an arc. Then the corresponding vertices $v_{1}, v_{2}, \ldots, v_{k}$ of $G$ are cut-vertices, and $G$ is the union of graphs whose layouts lie in the annular regions plus the innermost region: $G=G_{1} \cup G_{2} \cup \ldots \cup G_{k} \cup G_{k+1}$ where $G_{1}$ is the subgraph whose layout in L lies on $c_{1} \cup \operatorname{int}\left(c_{1}\right), G_{k+1}$ lies on $c_{k+1} \cup \operatorname{ext}\left(c_{k+1}\right)$, and for $i=2, \ldots, k, G_{i}$ lies on the annulus given by $c_{i-1} \cup c_{i} \cup\left\{\operatorname{int}\left(c_{i}\right) \cap \operatorname{ext}\left(c_{i-1}\right)\right\}$. Thus $G_{2}, \ldots, G_{k+1}$ are each planar. In addition for $i=2, \ldots, k G_{i}$ is 2-connected since each block of $G_{i}$ contains some vertices adjacent to $v_{i-1}$ and some to $v_{i}$. Thus the block-cutpoint tree for $G, \mathrm{BC}(G)$, contains a path of $2 k-1$ vertices, representing consecutively $v_{1}, G_{2}, v_{2}, \ldots, G_{k}, v_{k}$. What sorts of graphs are possible for $G_{1}$ and for $G_{k+1}$, and what additional tree structure in $\operatorname{BC}(G)$ is possible at the two ends of this path?

Consider $G_{1}$, laid out on $c_{1} \cup \operatorname{int}\left(c_{1}\right)$, with $c_{1}$ opened up to become an arc $a_{1}$ so that this is a PVG layout of $G_{1}$. If $G_{1}$ is planar, by Prop. 5 and its proof, $G_{1}$ can have at most one additional cut-vertex, not on the exterior face but represented by a long $\operatorname{arc} a^{*}$ at radius 1 . If there is no long $\operatorname{arc} a^{*}$ besides $a_{1}$, then $v_{1}$ may be attached to an arbitrary positive number of planar blocks. If there is a long arc $a^{*} \neq a_{1}$, then each block represented between $a^{*}$ and $a_{1}$ sees these two arcs and so there is only one block lying in this annular region. Inside and attached to $a^{*}$ may be any number $i_{a} \geq 0$ of 2-connected, planar graphs, but in any case, $\mathrm{BC}(G)$ has attached to the path-end $v_{1}$ either $i_{1}>0$ leaves or else one additional block vertex $b$, representing part or all of $G_{1}$, then a vertex for $a^{*}$ that is also adjacent to $i_{a}>0$ vertices of degree one. (Thus the latter case corresponds to having $v_{3}$ represented by $c_{1}$ and $v_{1}$ by $a^{*}$.) If $G_{1}$ is not planar, 
by Prop. 5 and Cor. 1 it is 2-connected so that the path of $\mathrm{BC}(G)$ is extended at $v_{1}$ by one additional vertex representing $G_{1}$.

The layout for the planar graph $G_{k+1}$ lies in the infinite region, $c_{k} \cup \operatorname{ext}\left(c_{k}\right)$. In this layout of $G_{k+1}$ the circle $c_{k}$ can be opened up to a long arc with empty interior to form a PVG layout; by Prop. $5 G_{k+1}$ has all its cut-vertices on a common face, the exterior face, and so can have arbitrarily many cut-vertices with arbitrarily many connected blocks, provided all cut-vertices lie on the infinite face. Thus attached to $v_{k}$ in $\mathrm{BC}(G)$ is any tree representing a planar graph with all cut-vertices, except possibly for $v_{k}$, on a common face. These remarks prove the necessity of Theorem 2 .

Lemma 1. Let $L$ be a layout of a PVG $G$ with $n$ vertices and with a long-arc pair at infinity or at the origin (or both). Then $L$ can be laid out as a CVG with a circle on the exterior face at radius $n$ or a circle about the origin at radius 1 (or both).

As noted in Section 3, a long arc at radius 1 or at $n$ can be extended to a full circle, changing no visibilities.

Proof. Proof of the sufficiency of Thm. 2, Suppose $G$ has $\mathrm{BC}(G)$ satisfying (1a) and $(2)$ so that $\mathrm{BC}(G)$ is $\left(b_{0}, e_{1}, e_{2}, \ldots, e_{2 k+1}, T\right)$ where for $i=1, \ldots, k$, each $e_{2 i-1}$ represents a cut-vertex $v_{i}$ of $G$, each $e_{2 i}$ represents a 2-connected planar graph, $b_{0}$ is a 2 -connected projective planar graph, and $T$ represents a plane graph with all cut-vertices on a face $F$. Such a graph embeds on the projective plane; in the layout each cut-vertex $v_{i}$ will be represented by a circle $c_{i}$.

By Prop. 4(iv) the projective planar subgraph of $G$ corresponding to $b_{0}$ has a PVG layout $L_{0}^{\prime}$ with the $\operatorname{arc} a_{1}$ representing $v_{1}$ in a long-arc pair at infinity with some neighbor of $v_{1}$. By Lemma $1 L_{0}^{\prime}$ can be changed to the CVG $L_{0}$ so that $a_{1}$ becomes a circle surrounding $L_{0}$. By Prop. 4(ii) the planar subgraph of $G$ corresponding to $e_{2}$ can be represented as a PVG $L_{1}^{\prime}$ with $a_{1}$, representing $v_{1}$, part of a long-arc pair at the origin and with $a_{2}$, representing $v_{2}$, part of a long-arc pair at infinity. By Lemma $1 L_{1}^{\prime}$ can be changed to the CVG $L_{1}$ so that $a_{1}$ and $a_{2}$ each become circles inside and surrounding $L_{1}$ respectively. Then $L_{1}$ is joined with $L_{0}$ by identifying the two copies of the circle $a_{1}$, placing $L_{1}$ wholly outside of $L_{0}$. This process of expansion can be repeated for $e_{4}, \ldots, e_{2 k}$. Finally by Prop. 4(i) $T$ can be laid out as a PVG with $v_{k}$ represented by $a_{k}$, part of a long-arc pair at the origin. Again by Lemma $1 a_{k}$ can be extended to a full circle inside of $T$ 's layout and can be identified with the circle representing $a_{k}$ on the exterior of the layout previously constructed. In this way $G$ is laid out.

If $\mathrm{BC}(G)$ satisfies (1b) and (2), it can be laid out similarly, only differing within $c_{1}$.

Since $v_{1}$ is incident with one or more planar blocks, we can lay these out in radial segments within $c_{1}$. Each planar block can be represented as a BVG with $v_{1}$ represented top-most and a neighbor bottom-most, then as a PVG via Prop. 2 and then inserted with $v_{1}$ 's arc as a subarc of $c_{1}$ within a distinct wedge of, say, $0 \leq \theta \leq \pi$, giving the desired visibilities. Thus in all cases the graph can be laid out as a CVG. 


\section{Concluding Thoughts}

It is clear that more complex graphs can be achieved in the polar visibility model by allowing visibility through the origin and diagonally across the boundary of a disc with antipodal points identified; call such a layout a doubly polar visibility layout and the resulting graphs doubly polar visibility graphs (DPVGs). These naturally lead to graphs that embed on the Klein bottle, the nonorientable surface of Euler characteristic 0. Analogous proofs to those given on the projective plane give the following results.

Proposition 6. a) A DPVG embeds on the Klein bottle.

b) If $G$ has a layout $L$ as a DPVG with no long arcs, then $G$ contains no cut-vertex.

c) If a 2-connected graph $G$ has an embedding on the Klein bottle, then $G$ is a $D P V G$ and has an equivalent doubly polar visibility layout. It seems that a DPVG that is neither a BVG nor a PVG can have at most two cut-vertices, represented by a long arc about the origin and at infinity.

Acknowledgements. The author wishes to thank Alexandru Burst, Alice Dean, Michael McGeachie, Bojan Mohar, William Owens, and Stan Wagon for useful conversations and insightful examples concerning this work.

\section{References}

1. Bose, P., Dean, A., Hutchinson, J., Shermer, T. On rectangle visibility graphs In: North, S. (ed.): Proc. Graph Drawing '96. Lecture Notes in Computer Science, Vol. 1190. Springer, Berlin (1997) 25-44

2. Chang, Y-W., Jacobson, M. S., Lehel, J., West, D. B. The visibility number of a graph (preprint)

3. Dean, A., Hutchinson, J. Rectangle-visibility representations of bipartite graphs Discrete Applied Math. 75 (1997) 9-25

4. ----- Rectangle-visibility layouts of unions and products of trees J. Graph Algorithms and Applications 2 (1998) 1-21

5. Dean, A. Bar-visibility graphs on the Möbius Band In: Marks, J. (ed.): Proc. Graph Drawing 2000. (to appear)

6. Hutchinson, J., Shermer, T., Vince, A. On Representations of some Thickness-two graphs Computational Geometry, Theory and Applications 13 (1999) 161-171

7. Mohar, B. Projective planarity in linear time J. Algorithms 15 (1993) 482-502

8. Mohar, B., Rosenstiehl, P. Tessellation and visibility representations of maps on the torus Discrete Comput. Geom. 19 (1998) 249-263

9. Mohar, B., Thomassen, C. Graphs on Surfaces Johns Hopkins Press (to appear)

10. O'Rourke, J. Art Gallery Theorems and Algorithms Oxford University Press, Oxford (1987)

11. Tamassia, R., Tollis, I. G. A unified approach to visibility representations of planar graphs Discrete and Computational Geometry 1 (1986) 321-341

12. --- Tesselation representations of planar graphs In: Medanic, J. V., Kumar, P. R. (eds.): Proc. 27th Annual Allerton Conf. on Communication, Control, and Computing. (1989) 48-57 
13. ----- Representations of graphs on a cylinder SIAM J. Disc. Math. 4 (1991) 139-149

14. Thomassen, C. Planar representations of graphs In: Bondy, J. A., Murty, U. S. R. (eds.): Progress in Graph Theory. (1984) 43-69

15. West, D. Introduction to Graph Theory Prentice Hall, Upper Saddle River, NJ (1996)

16. White, A. Graphs, Groups and Surfaces revised ed. North-Holland, Amsterdam (1984)

17. Wismath, S. Characterizing bar line-of-sight graphs In: Proc. 1st Symp. Comp. Geom. ACM (1985) 147-152 